\title{
Identification and analysis of cutting force coefficients in the helical milling process
}

\author{
Haiyan WANG *, Jianyu WANG *, Jinming ZHANG *, Kexin TAO * and Dongxu WU * \\ ${ }^{*}$ School of Control Engineering, Northeastern University at Qinhuangdao, \\ 143 Taishan Road, Qinhuangdao, 066004, China \\ E-mail: hywang16@126.com
}

Received: 5 August 2019; Revised: 13 February 2020; Accepted: 9 March 2020

\begin{abstract}
Cutting forces are often used to optimize cutting parameters and analyze cutting characteristics of the tool. Accurate prediction of cutting forces is critical to understand deeply of the cutting process, especially for helical milling of the difficult-to-cut materials. Generally, the cutting force coefficients are not affected by the cutting parameters in the end milling process, but due to the special cutting mechanism of helical milling, the change of the cutting force coefficients must be carefully studied. However, the identification of cutting force coefficients is very important to simulate cutting forces accurately, and the relationship between cutting force coefficient and cutting parameters needs to be carefully considered. To analyze the change of cutting forces, this paper respectively proposes linear and nonlinear cutting force models applied for the helical milling process, then according to the helical milling experiments, cutting coefficients are identified using an inverse method through instantaneous force in the linear model, at the same time, the coefficients are identified by similar average force method in the nonlinear model, simulated three-direction cutting forces under two different situations are compared to the experimental results. The relationship between cutting force coefficients and cutting parameters is analyzed in detail, the linear and nonlinear models as well as the fitting relations of different cutting force coefficients are compared. The aim of this paper is to study the influence of cutting parameters on the cutting coefficients, thus create a model to predict reliably the cutting force with different cutting parameters.
\end{abstract}

Keywords : Helical milling, CFRP, Cutting force, Cutting coefficients, Linear, Nonlinear

\section{Introduction}

As a kind of new hole-making technology, helical milling process has arisen attention widely. In the cutting process, the tool proceeds a helical path while rotates around its own axis, thus the movement of tool comprises both the tangential cutting and the axial cutting, the frontal cutting edges of tool mainly produce axial cutting force, but the peripheral edges not only produce radial cutting forces but also axial forces (Iyer et al.,2007;Pereira et al.,2017), these two-direction cutting forces will influence cutting quality in different degree, so it is very important to setup the cutting force models. Wang et al. $(2012,2016)$ first gave the linear cutting force model in the helical milling of titanium alloy. The results showed that the established linear model could accurately reflect the variation of cutting forces. At the same time, the cutting force model in the helical milling of CFRP considering the direction of fiber lamination and cutting angle was also established. Li et al. (2014) considered the influence of both peripheral edge and frontal edge on cutting mechanism and cutting force, proposed a dynamic model of cutting force for helical milling process, revealing the influence of feed speed on cutting force model. Liu et al. (2012) established the cutting force model in helical milling process on the basis of helical feed, spindle speed, axial and radial cutting depth, described the cutting forces of 
Haiyan Wang, Jianyu Wang, Zhang, Tao and Wu,

Journal of Advanced Mechanical Design, Systems, and Manufacturing, Vol.14, No.1 (2020)

peripheral edge and frontal edge along the helical feed path through considering the tangential and axial motion of the cutter, and verified it using the helical milling experiment of titanium alloy. Rey et al. (2016) established a mechanical cutting force model related to the cutter geometry and cutting parameters and instantaneous chip thickness, so that the quality of hole-making can be improved by controlling the cutting force. Sadek et al. (2012) proposed a cutting force model based on fracture mechanics, and found that the main factors causing the outlet delamination of CFRP material were the axial load distribution. There are more and more researches on cutting force modeling in the helical milling process, and through the study of related literature, it can be known that the identification of cutting force coefficient is very critical.

There were different options to obtain the specific cutting coefficients from the experimental results, among them, the most were based on average force method. However, other methods were also used to study the identification of cutting force coefficient, which had become hot issues (Azeem et al.,2004; Adetoro and Wen,2009;Wan et al.,2010; Schwenzer et al.,2019). Budak et al.(1996)firstly presented mechanics method based on oblique cutting model, the main purpose was to reduce a large number of experimental requirements after changing the tool, the identified milling coefficients had been experimentally verified. Gradisek et al. (2004) resented mechanistic identification of specific cutting and edge coefficients for a general end mill using average force methods. Wang et al. (2012)used an improved method to calculate cutting coefficients and edge coefficients in the milling process, supposed that milling coefficients were time invariant for small variation of feed rate. Gonzalo et al. (2010, 2019) presented the specific coefficients depended on tool-material couple and the geometry of the tool, and verified through virtual experiments using a finite element method, in addition, the instantaneous cutting force coefficients were identified, and the accuracy of the cutting force coefficients was solved by the least square method. Kalla et al. (2010) utilized ANN to accurate predict of specific cutting energies of unidirectional composites in the general milling process. Adem et al. (2015) presented the linear and nonlinear mathematical force models for an end-milling process using two kind of tools. The result showed that optimization technique was better than average force method, and nonlinear mathematical force model was more precise than linear model. Shang et al. (2018) also established a cutting force model for the helical milling process, and identified the cutting force coefficient by using the average force method, the accuracy of cutting force model under different cutting depth conditions was analyzed precisely.

Usually, there are two kinds of cutting force models used to analyze in the end milling process, one is the linear model, which suggests cutting forces have a linear relation to the uncut chip thickness.

$$
F_{i}=\left(K_{i c} \cdot h+K_{i e}\right) \cdot z \quad i \in t, r, a
$$

where $F_{i}$ is any analytical cutting force components in tangential, radial and axial directions, $K_{i c}$ is the cutting coefficient for linear force model, $K_{i e}$ is the edge coefficient, $h$ is the chip thickness, $z$ is the axial position of the tool. The other is the nonlinear model, which assumes cutting forces have an exponential relationship with the uncut chip thickness as follows

$$
F_{i}=K_{i} \cdot z \cdot h^{1-m_{i}} \quad i \in t, r, a
$$

where $K_{i}$ is the cutting coefficient; $m_{i}$ is the index. As such, the cutting force coefficients are major parameters in the cutting force model, and their accurate identification has a great influence on the research of cutting process. However, accurate determination of these empirical force coefficients over a wide range of cutting conditions was rather time consuming, since it required a large number of experiments with varying parameters to be included in the experiment design, so establishment of the relationship between the cutting force coefficient and cutting parameters was even more critical in the model of cutting forces (Azeem et al.,2004). While in the helical milling process, cutter needs to complete axial feed with $0.1-0.2 \mathrm{~mm}$ per orbital revolution continually, as shown in Fig.1, the change of cutting forces and cutting coefficients are relatively complex, so this paper will study cutting force models in the helical milling of CFRP from the angle of linear and nonlinear, identify special cutting coefficients under different condition, and analyze the difference 
of the force coefficients in the linear and nonlinear model, complete the comparison of linear and nonlinear model precision, so as to create a model able to reliably predict the cutting force with different cutting parameters.

\section{Modeling of cutting forces in helical milling process}

\subsection{Establishment the coordinate systems}

In the helical milling process, tool diameter is smaller than hole diameter so that chip can be eliminated smoothly. In order to analyze the moving state of the tool correctly, two reference coordinate systems are set up first: tool reference frame and workpiece reference frame. The original point of tool reference frame (the tool center location) is $C$, then the cutter coordinate system $x_{\mathrm{c}} C y_{\mathrm{c}}$ is established; the original point of workpiece reference frame is (hole center) $O$, then the workpiece coordinate system $x O y$ is also set up, as shown in Fig.1.

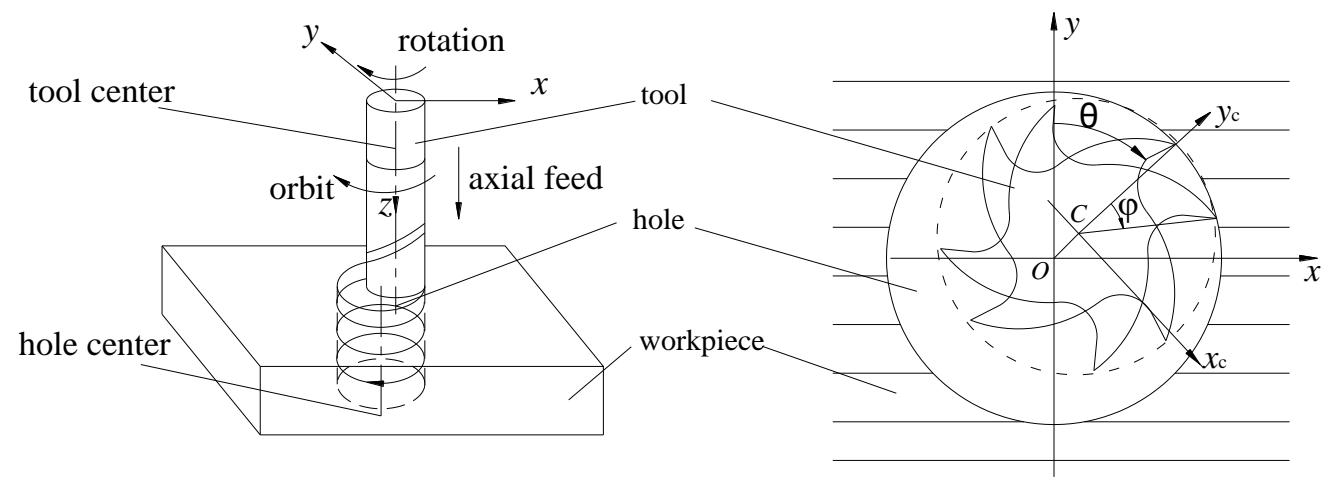

Fig.1 Helical milling kinematics

\subsection{Modeling of cutting forces}

According to the differential thought of the cutting edge, the axial direction of the cutting edge cutting into the workpiece is divided into a certain number of micro-element edges, so that each disc is considered as an orthogonal cutting edge, and the micro-element tangential, radial and axial cutting forces can be expressed by the product of the cutting force coefficient and the undeformed chip area. That is to say, the micro element models of cutting force in the tool coordinate system need to be firstly established.

\subsubsection{Linear model}

Generally, axial force is not or little considered in the end milling process, but the effect of axial force is particularly important in the helical milling process, thus three - way cutting forces are to be considered. The linear cutting force model was originally proposed by Tlusty and MacNeil (1975), then subsequently developed by Altintas $(1991,1996,1996)$, then the micro-element tangential, radial and axial cutting forces acting on the $i$ th cutter tooth could be expressed as

$$
\left\{\begin{array}{l}
d F_{t i}(\varphi, z)=K_{t c} h_{i}(\varphi, z) d z+K_{t e} d z \\
d F_{r i}(\varphi, z)=K_{r c} h_{i}(\varphi, z) d z+K_{r e} d z \\
d F_{a i}(\varphi, z)=K_{a c} h_{i}(\varphi, z) d z+K_{a e} d z
\end{array}\right.
$$

where $d F_{t}, d F_{r}$ and $d F_{a}$ are the micro-element force in tangential, radial and axial directions, and $K_{t c}, K_{r c}$ and $K_{a c}$ are cutting force coefficient in tangential, radial and axial directions, $K_{t e} 、 K_{r e}$ and $K_{a e}$ are edge coefficient in tangential, radial and axial directions, $h_{i}$ is the undeformed chip thickness, $d z$ is the micro-element cutting depth, $h_{i}(\varphi, z) d z$ is the area of undeformed chip of micro cell, and undeformed chip thickness can be written as 


$$
h_{i}(\varphi, z)=s_{t} \sin \varphi_{i}(t)
$$

where $S_{t}$ is the feed rate per tooth, $\varphi_{i}$ is the cutter angle in the tool coordinate system, at each instantaneous position of the tool, the rotation angle can be expressed as

$$
\varphi_{i}(t)=\varphi_{1}(t)+(i-1) * 2 \pi / N-2 * z * \tan \beta / D_{T}
$$

where $N$ is the number of cutting teeth, $\beta$ is the helix angle of the tool, $D_{\mathrm{T}}$ is the tool diameter.

\subsubsection{Nonlinear model}

For helical milling process, the relationship between cutting force and undeformed chip thickness is more complex, so it is very necessary to establish a nonlinear cutting force model. Usually, supposed that three main cutting coefficients are the function of the undeformed chip thickness:

$$
\left\{\begin{array}{l}
K_{t c}=K_{t} h^{-p} \\
K_{r c}=K_{r} h^{-q} \\
K_{a c}=K_{a} h^{-m}
\end{array}\right.
$$

where $K_{t}, K_{r}$ and $K_{a}$ are cutting force coefficients in tangential, radial and axial direction for the nonlinear force model, $p 、 q$ and $m$ are tangential, radial and axial indexes, and supposed that edge forces are zero in Eq.(3), namely

$$
K_{t e}=K_{r e}=K_{a e}=0
$$

Even if the cutting force coefficients under certain condition are identified, they must be changed according to the change of undeformed chip thickness. The Eq.(6) needs to be substituted into Eq.(3), thus the nonlinear cutting force model is established as follows:

$$
\left\{\begin{array}{l}
d F_{t i}(\varphi, z)=K_{t} h_{i}^{1-p}(\varphi, z) d z \\
d F_{r i}(\varphi, z)=K_{r} h_{i}^{1-q}(\varphi, z) d z \\
d F_{a i}(\varphi, z)=K_{a} h_{i}^{1-m}(\varphi, z) d z
\end{array}\right.
$$

\subsection{Coordinate transformation}

Whether it is a linear model or a nonlinear model, the cutting forces in the tool coordinate system need to be converted into the workpiece coordinate system, so as to verify through the experimental values. The cutting forces in the workpiece coordinate system are the forces measured by the dynamometer in the actual cutting process, which is consistent with the direction of the machine tool coordinate system. The relationship between two coordinate systems is illustrated as follow:

$$
\left[\begin{array}{l}
d F_{x i}\left(\theta, \varphi_{i}, z\right) \\
d F_{y i}\left(\theta, \varphi_{i}, z\right) \\
d F_{z i}\left(\theta, \varphi_{i}, z\right)
\end{array}\right]=\left[\begin{array}{ccc}
\cos \theta & \sin \theta & 0 \\
-\sin \theta & \cos \theta & 0 \\
0 & 0 & 1
\end{array}\right] \cdot\left[\begin{array}{ccc}
\cos \alpha & 0 & 0 \\
0 & \cos \alpha & 0 \\
-\sin \alpha & -\sin \alpha & 1
\end{array}\right] \cdot\left[\begin{array}{ccc}
-\cos \varphi_{i} & -\sin \varphi_{i} & 0 \\
\sin \varphi_{i} & -\cos \varphi_{i} & 0 \\
0 & 0 & -1
\end{array}\right] \times\left[\begin{array}{l}
d F_{t i}\left(\varphi_{i}, z\right) \\
d F_{r i}\left(\varphi_{i}, z\right) \\
d F_{a i}\left(\varphi_{i}, z\right)
\end{array}\right]
$$

where $\theta$ is the tool position in the workpiece coordinate system, $\alpha$ is the pitch angle, which can be expressed as

$$
\alpha=\arctan \left(a /\left(\pi *\left(D_{H}-D_{T}\right)\right)\right)
$$

$D_{\mathrm{H}}$ is the hole diameter, $a$ is the axial depth of cut. 
According to the characteristics of tool movement in the helical milling process, as shown in Fig.2, the special tool rotates and makes a small step forward in the direction of orbital revolution, when the tool rotates, supposed that $Q$ is entry point, $P$ is the exit point, then at any time, the rotational angle of the tool must be lied in

$$
0 \leq \varphi_{i} \leq \pi
$$

Simultaneously, the tool needs to be completed revolution around the hole center, so

$$
0 \leq \theta \leq 2 \pi
$$

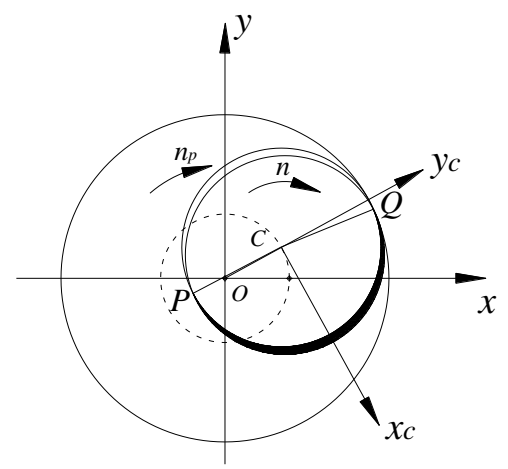

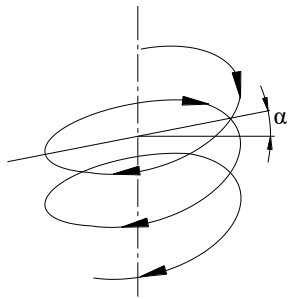

tool center path

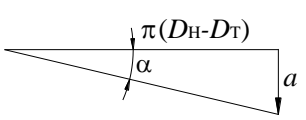

Fig.2 Tool movement trajectory

Supposed that $z$ is the thickness of the plate, finally, total cutting forces on the $i$ th cutting edge can be illustrated as

$$
\left\{\begin{array}{l}
F_{x i}\left(\varphi_{i}, \theta\right)=\int_{z 1}^{z 2} d F_{x i}\left(\varphi_{i}, \theta, z\right) \\
F_{y i}\left(\varphi_{i}, \theta\right)=\int_{z 1}^{z 2} d F_{y i}\left(\varphi_{i}, \theta, z\right) \\
F_{z i}\left(\varphi_{i}, \theta\right)=\int_{z 1}^{z 2} d F_{z i}\left(\varphi_{i}, \theta, z\right)
\end{array}\right.
$$

$F_{x i}, F_{y i}$ and $F_{z i}$ are micro-element force in $x$-direction, $y$-direction and $z$-direction $(\mathrm{N})$. Analyzing all the cutter teeth participating in the helical milling process, the instantaneous cutting force in the global workpiece coordinate system can be expressed as

$$
\left\{\begin{array}{l}
F_{x}(\varphi, \theta)=\sum_{i=1}^{N} g\left(\varphi_{i}\right) F_{x i}(\varphi, \theta) \\
F_{y}(\varphi, \theta)=\sum_{i=1}^{N} g\left(\varphi_{i}\right) F_{y i}(\varphi, \theta) \\
F_{z}(\varphi, \theta)=\sum_{i=1}^{N} g\left(\varphi_{i}\right) F_{z i}(\varphi, \theta)
\end{array}\right.
$$

where $F_{x}, F_{y}$ and $F_{z}$ are cutting forces in $x, y$ and $z$-directions, $g\left(\varphi_{i}\right)$ is a specific parameter used to indicate whether the cutter tooth is in the cutting state, when the cutter tooth is in the cutting state, $g\left(\varphi_{i}\right)=1$, and conversely, when the cutter tooth is not in the cutting state or out of the cutting state, $g\left(\varphi_{i}\right)=0$.

\subsection{Identification of instantaneous cutting coefficients}

In order to understand the effects of cutting parameters on the cutting force coefficients, the instantaneous force method is used to identify cutting force coefficients according to each kind of cutting condition, without thinking the 
effect of tool wear on the cutting force separately, therefore the cutting force coefficients under different cutting conditions are calculated based on the recognition of simulated and measured forces in the time domain. Equation(14) needs to be converted into the function relation between cutting force coefficient and corresponding parameter, the left part of the Eq.(15) is any instantaneous three-direction simulated cutting forces, which could be fitted theoretically through Eq.(14), and it can be represented as a function between cutting coefficients and relevant cutting parameters: cutting time $t$, orbital angle $\theta$, rotational angle $\varphi$, and undeformed chip thickness $h$ and cutting depth $a$. In fact, it is a function of cutting force and cutting parameters, cutting time and cutting force coefficients.

$$
\left\{\begin{array}{l}
F_{x} \\
F_{y} \\
F_{z}
\end{array}\right\}=[A(t, \theta, \varphi, h, a)]\left\{\begin{array}{l}
K_{t c} \\
K_{t e} \\
K_{r c} \\
K_{r e} \\
K_{a c} \\
K_{a e}
\end{array}\right\}
$$

where $A$ represents the transformation relation of related functions, $\varphi$ and $\theta$ can be obtained by the following formulas:

$$
\varphi=\omega t \quad \theta=\omega_{1} t
$$

then corresponding angle speeds are written as, respectively

$$
\omega=2 \pi n / 60 \quad \omega_{1}=2 \pi n_{p} / 60
$$

where $\omega$ is the rotational angle speed, $\omega_{1}$ is the orbital angle speed, and $n$ is the spindle speed, $n_{p}$ is the orbital speed. As long as the rotational and orbital speeds of the tool are known, the rotation angle and orbital angle of the tool at any time will be known. For the cutting force measured in the experiment, due to the complexity of tool movement, the cutting force value will change at any time, so more time points need to be selected to verify, the following formula also exists for any time

$$
\left\{\begin{array}{l}
F_{x} \\
F_{y} \\
F_{z}
\end{array}\right\}=[A(t, \theta, \varphi, h, a)]\left\{\begin{array}{l}
K_{t c} \\
K_{t e} \\
K_{r c} \\
K_{r e} \\
K_{a c} \\
K_{a e}
\end{array}\right\}
$$

Namely, supposed that corresponding experimental and simulated cutting forces are same, the cutting force coefficients can be solved from Eq.(19).

$$
\left\{\begin{array}{c}
F_{x} \\
F_{y} \\
F_{z}
\end{array}\right\}^{\text {Experimental }}=\left\{\begin{array}{c}
F_{x} \\
F_{y} \\
F_{z}
\end{array}\right\}^{\text {Simulated }}=[A(t, \theta, \varphi, h, a)]\left\{\begin{array}{l}
K_{t c} \\
K_{t e} \\
K_{r c} \\
K_{r e} \\
K_{a c} \\
K_{a e}
\end{array}\right\}
$$


There will be three-direction cutting forces measured through experiments at time $t_{1}$, at next time $t_{2}$, namely, corresponding different time will have the corresponding cutting forces, thus a time period is selected as the basis for analysis. In this way, supposed that the simulated force and the experimental values are equal in several discrete times, a constrained least square fitting method can be used, simultaneously in order to improve the results, different weights and constraints could be selected for analysis to eliminate the influence of random errors. The objective is to minimize the following equation:

$$
\sum_{n}\left\|y_{i}-f_{i}\left(t, K_{t c}, K_{r c}, K_{a c}, K_{t e}, K_{r e}, K_{a e}\right)\right\|_{D}^{2}+\left\|m-m_{p}\right\|_{M}^{2}
$$

where $y_{i}$ is the measured force, $f_{i}$ is function, which represents the simulated force, $m$ and $m_{p}$ are the iterative solution vector and initial estimation of cutting coefficients values, $D$ and $M$ are weighting factors. The discrete time and its corresponding measured force are substituted into the Eq.(20), the constrained least square method is used to solve the instantaneous cutting coefficients, and the cutting force coefficients under different cutting conditions can be identified.

\subsection{Identification of average cutting coefficients}

Generally, the cutting force coefficients can also be identified by calculating the average milling force of the cutter within an orbital period. Firstly, the average milling forces of three directions in the workpiece coordinate system are calculated. Considering the non-linear relationship, the measured cutting force should be processed. Supposed that $F_{\mathrm{Aq}}$ represents the three-direction cutting forces measured under certain cutting conditions. The natural logarithm of the measured cutting forces is fitted to the linear relation of the logarithm of feed rate

$$
\ln \left(F_{A q}\right)=B_{q} \ln s_{t}+A_{q} \quad q=x, y, z
$$

where $F_{A q}$ is the fitted forces, $B_{q}$ and $A_{q}$ are the fitted parameters, the analytical Eq.(14) is also processed accordingly.

$$
\ln \bar{F}_{q}=\ln \frac{1}{\varphi_{p}\left(\theta_{2}-\theta_{1}\right)} \int_{0}^{\pi} \int_{0}^{\pi} F_{q}(\varphi, \theta) d \varphi d \theta \quad(q=x, y, z)
$$

According to the relationship among all the above formulas, thus

$$
\left\{\begin{array}{l}
p=1-B_{x} \\
q=1-B_{y} \\
m=1-B_{z}
\end{array}\right.
$$

Thus the corresponding exponents $p 、 q$ and $m$ in Eq. (8) are all calculated respectively. At the same time, Eq. (21) in the linear model is converted into Eq. (22), the integral result of the remaining parts is equal to $e^{A q}$, and finally, all the cutting force coefficients and indexes for the nonlinear force model can be calculated.

\section{Helical milling experiments}

Cutting experiment in helical milling of unidirectional CFRP with $0^{\circ}$ lamination was carried out on the five-axis machining center, as shown in Fig.3. The $x / y / z$ three-direction cutting forces were measured respectively. 


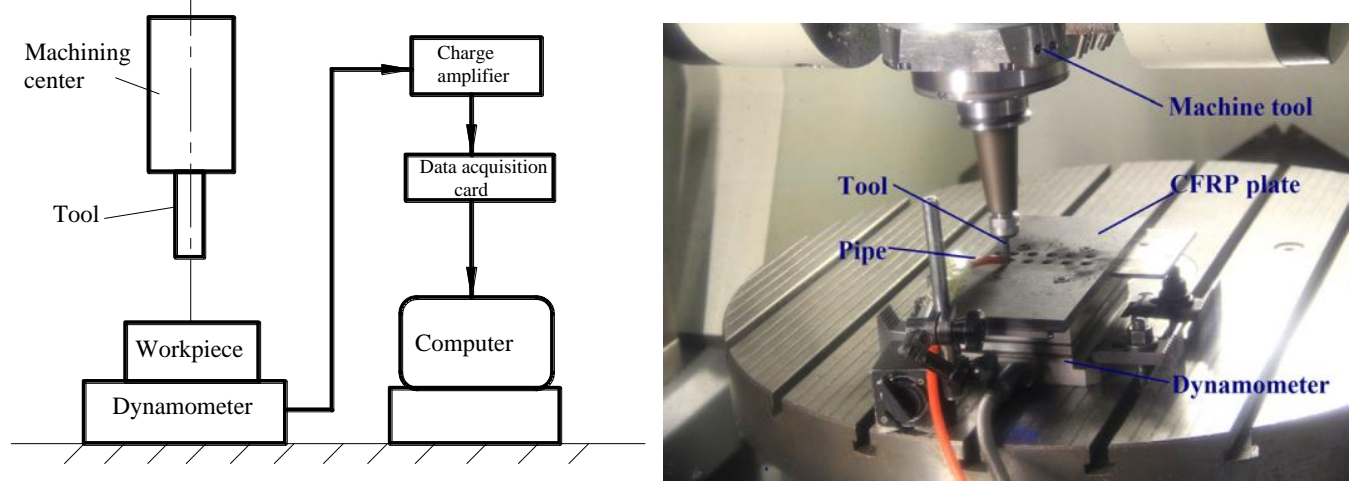

Fig.3 Experiment setup

Tool used was four teeth special helical milling tool with $6 \mathrm{~mm}$ diameter, coating materials was TiAlN, single factor experiment using to identify of cutting force coefficient was arranged as follows: the spindle rotation speed was 3000 $\mathrm{rpm}$, axial cutting deep was $0.15 \mathrm{~mm} / \mathrm{rev}$, feed rates were $0.03,0.04,0.05,0.06 \mathrm{~mm} /$ tooth, and in order to verify the accuracy of the cutting force coefficients, full factor experiments were also carried out, and the cutting parameters were illustrated in Table 1.

Table 1 Full factor cutting parameters in the helical milling of CFRP

\begin{tabular}{c|c|c|c}
\hline \hline \multirow{2}{*}{ Factors } & \multicolumn{3}{|c}{ Levels } \\
\cline { 2 - 4 } & 1 & 2 & 3 \\
\hline Spindle speed $n /(\mathrm{rpm})$ & 4000 & 5000 & 6000 \\
Feed rate $s_{\mathrm{t}} /(\mathrm{mm} / \mathrm{tooth})$ & 0.02 & 0.04 & 0.06 \\
Depth of cut $a /(\mathrm{mm} / \mathrm{rev})$ & 0.1 & 0.15 & 0.2 \\
\hline
\end{tabular}

In order to verify the accuracy of cutting force coefficients, the measured cutting force under several conditions are selected as the basis of analysis.

\section{Results and discussions}

\subsection{Modification of cutting force model}

Cutting force model needed to meet helical milling machining characteristics, if the material was anisotropic materials (Brinksmeier et al., 2011). It needs to consider the influence of fiber orientation in the machining of CFRP, although fiber layer direction remains constant in the whole cutting process, due to the rotation and orbital revolution of the tool, fiber cutting angle will change real-time in the different position of hole-making, thus fiber orientation will directly affect the size and fluctuation of three-way cutting forces, so the original model needs to be modified. Considering the actual situation, $x$ axis is the direction of fiber layer, and $y$ axis is perpendicular to the fiber layer, as shown in Fig.4.

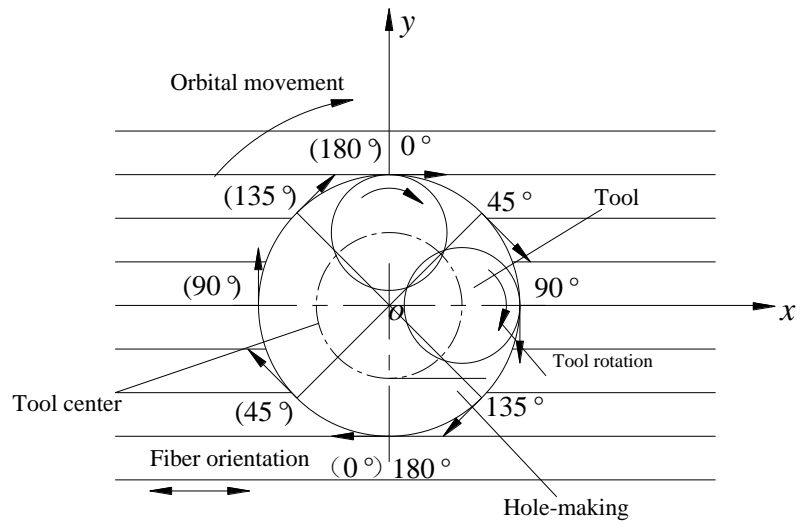

Fig. 4 Definition of fiber cutting angle 
In the helical milling of CFRP, different fiber cutting angles will appear at different cutting positions due to the influence of tool movement. As shown in Fig. 4, the fiber cutting angle is defined as the angle between the cutter feed direction and the fiber layering direction. Due to the fiber orientation is zero degree, fiber cutting angle will be changed from 0 to $180 \mathrm{deg}$ second times in one orbital revolution, therefore, the cutting forces will fluctuant accordingly, especially in axial direction. Thus, the influence of fiber cutting angle has been considered in the axial force

$$
F_{z}(\varphi, \theta)=\sum_{i=1}^{N} g\left(\varphi_{i}\right) * F_{z i}(\varphi, \theta) * \sin (2 * \theta)
$$

When the spindle speed is $5000 \mathrm{rpm}$, feed rate is $0.02 \mathrm{~mm} / \mathrm{t}$, and the axial depth of cut is $0.2 \mathrm{~mm} / \mathrm{r}$, the measured three-way cutting forces in the helical milling of CFRP are shown in Fig.5 (a). It can be seen that peak forces in the $x$ and $y$ direction in helical milling of CFRP are not same. Through the analysis of all the measured cutting forces, it can be found that at any moment, the peak value in $x$-direction is greater than $y$-direction, so a coefficient of 1.2 will be introduced in the cutting force model in $x$ direction to show the anisotropic of CFRP.

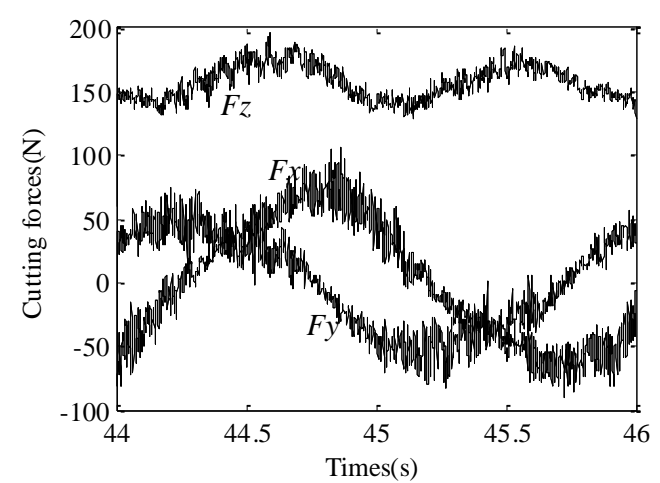

(a) Measured values

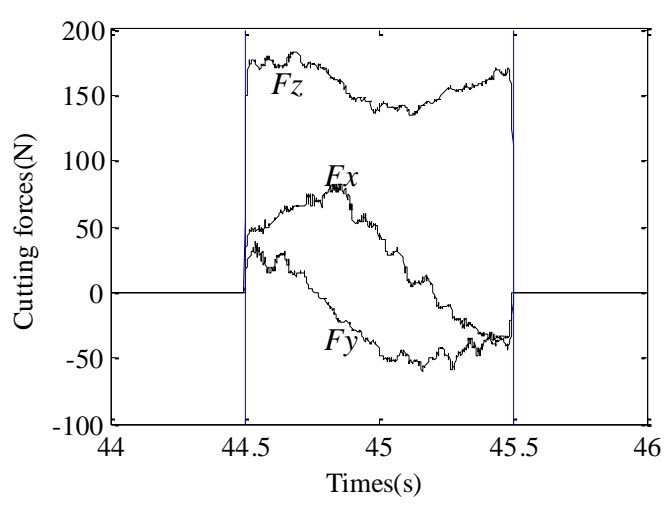

(b) Selected zone

Fig.5 Measured and selected zones to obtain local forces

In addition, because of axial feed of tool in the helical milling process, an indentation force $F_{f a}$ needs to be added in the axial direction in Eq.(24). The indentation force is caused by the frontal edge, and can be calculated as follows:

$$
F_{f a}=\left(K_{f c} * f_{a}+K_{f e}\right) * D_{T} / 2
$$

where $K_{f c}$ is the axial cutting coefficient, $K_{f e}$ is the axial edge coefficient, $f_{a}$ is the axial feed rate per tooth.

$$
f_{a}=s_{t} * \tan \alpha
$$

Therefore, the modified three-direction cutting forces are expressed as

$$
\left\{\begin{array}{c}
F_{x}(\varphi, \theta)=1.2 * \sum_{i=1}^{N} g\left(\varphi_{i}\right) F_{x i}(\varphi, \theta) \\
F_{y}(\varphi, \theta)=\sum_{i=1}^{N} g\left(\varphi_{i}\right) F_{y i}(\varphi, \theta) \\
F_{z}(\varphi, \theta)=\sum_{i=1}^{N} g\left(\varphi_{i}\right)\left(F_{z i}(\varphi, \theta) * \sin (2 * \theta)+F_{f a}\right)
\end{array}\right.
$$

\subsection{Identification results of cutting force coefficients}

For the nonlinear cutting force model, three cutting force coefficients and three indexes are identified through 
Haiyan Wang, Jianyu Wang, Zhang, Tao and Wu,

Journal of Advanced Mechanical Design, Systems, and Manufacturing, Vol.14, No.1 (2020)

similar average force method, and the cutting coefficients of the frontal edge in Eq.(24) are identified through linear fitted methods. The results are illustrated in Table 2.

Table 2 Nonlinear cutting coefficients and exponents in helical milling of CFRP

\begin{tabular}{c|c|c|c|c|c|c|c|c}
\hline \hline Symbols & $K_{t}\left(\mathrm{~N} / \mathrm{mm}^{2}\right)$ & $K_{r}\left(\mathrm{~N} / \mathrm{mm}^{2}\right)$ & $K_{a}\left(\mathrm{~N} / \mathrm{mm}^{2}\right)$ & $p$ & $q$ & $m$ & $K_{f c}\left(\mathrm{~N} / \mathrm{mm}^{2}\right)$ & $K_{f e}(\mathrm{~N} / \mathrm{mm})$ \\
\hline Values & 3781.7 & 3220.362 & 349.99 & 0.04 & 0.05 & 0.5 & 84956 & 25.86 \\
\hline
\end{tabular}

In order to identify the cutting coefficients using instantaneous force method, take the three-direction cutting forces in Fig.5 as example, the scope which can reflect of the cutting force peak value is selected to analyze, as shown in Fig.5(b). The instantaneous force coefficients can be identified through Eq.19-20 under different cutting parameters. Take the shear force coefficients $\left(K_{\mathrm{tc}}\right)$ as the example, the change of shear coefficients with the change of cutting parameters are shown in Fig.6. It can be seen the instantaneous shear coefficients are not constant in the helical milling process, the shear coefficients change with the change of cutting parameters, and changing trend is obvious, in addition, the influence of cutting parameters on cutting force coefficient is not same, the cutting coefficients increase with the increase of spindle speed, however decrease with the increase of the feed rate and cutting depth.
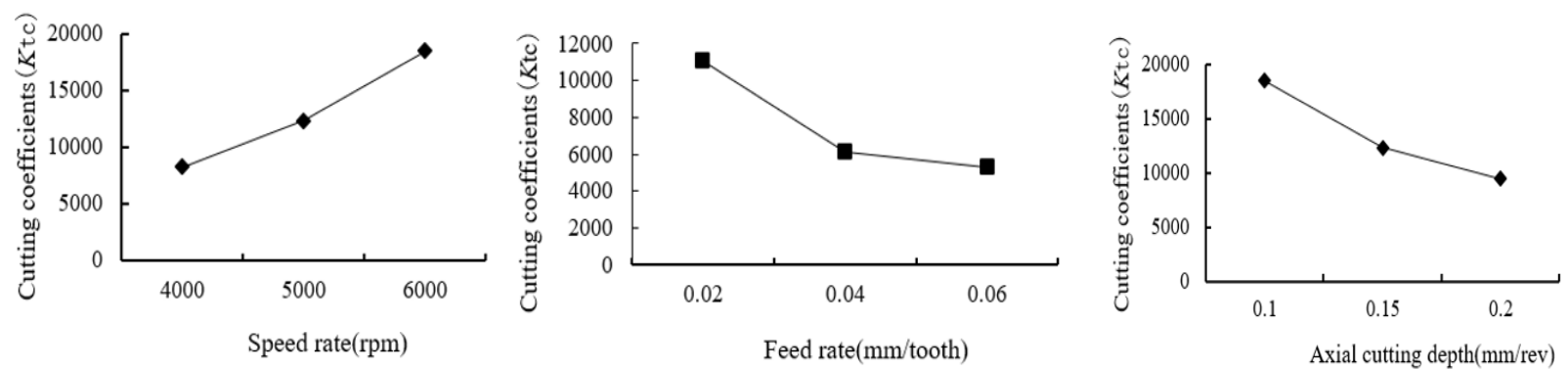

Fig.6 Relationship between cutting coefficients $\left(K_{\mathrm{tc}}\right)$ and cutting parameters

\subsection{Comparison of simulated and experimental cutting forces}

The simulated and experimental three direction cutting forces under different cutting parameters are illustrated in Fig.7, the related cutting parameters are shown in Table 3. As shown in Fig.7, the red lines are the simulated nonlinear cutting forces, while the blue lines are the simulated linear cutting forces, and wavy lines are experimental results.

Table 3 Cutting parameters corresponding to the fitting figures

\begin{tabular}{c|c|c|c}
\hline \hline Figure & Spindle speed (rpm) & Feed rate(mm/tooth) & Axial cutting depth (mm/rev) \\
\hline Fig.7 (a) & 3000 & 0.05 & 0.15 \\
Fig.7 (b) & 4000 & 0.04 & 0.15 \\
Fig.7 (c) & 6000 & 0.06 & 0.1 \\
Fig.7 (d) & 5000 & 0.06 & 0.2 \\
\hline
\end{tabular}

It can be seen that the fitting results of linear and nonlinear models are all good in Fig.7(a), although there are some differences to the fitting effect of different models. Compared to the linear cutting force, nonlinear cutting force in the $x$ and $y$ direction will be smaller, $z$ direction is not affected, the reason may be due to the friction is ignored in the nonlinear model, the maximum cutting force will reduce without influencing the actual change of cutting forces. While in the building of the nonlinear force model, assuming that the cutting force coefficients are the function of undeformed chip thickness, namely, cutting force coefficients are the function of feed rates, thus the change of feed rate will not influence the cutting forces.

But for Fig.7(b), axial cutting depth keeps unchangeable relative to the identification process, the spindle speeds 
Haiyan Wang, Jianyu Wang, Zhang, Tao and Wu,

Journal of Advanced Mechanical Design, Systems, and Manufacturing, Vol.14, No.1 (2020)

change from 3000rpm to 4000rpm, the fitting effect is good as Fig.7(a); while for Fig.7(c), cutting depths and spindle speeds are all changed, cutting depths decrease from $0.15 \mathrm{~mm} / \mathrm{r}$ to $0.1 \mathrm{~mm} / \mathrm{r}$, and spindle speeds increase from $3000 \mathrm{rpm}$ to 6000rpm, it can be seen that the max cutting forces in the $x$ and $y$ directions of the nonlinear model are smaller than the linear model, cutting forces in $z$ direction are larger than linear model, but the fitted effect is accepted according to the experimental forces. While in the Fig.7 (d), both the cutting depths and spindle speeds increase relative to the identified process, the cutting depths increase from $0.15 \mathrm{~mm} / \mathrm{r}$ to $0.2 \mathrm{~mm} / \mathrm{r}$, spindle speeds increase from $3000 \mathrm{rpm}$ to 5000rpm, the results are different to Fig.7(c), the max forces in the $x$ and $y$ directions in the nonlinear model are same as the linear model, cutting forces in $z$ direction is smaller than linear model, the fitted effect is relatively good.

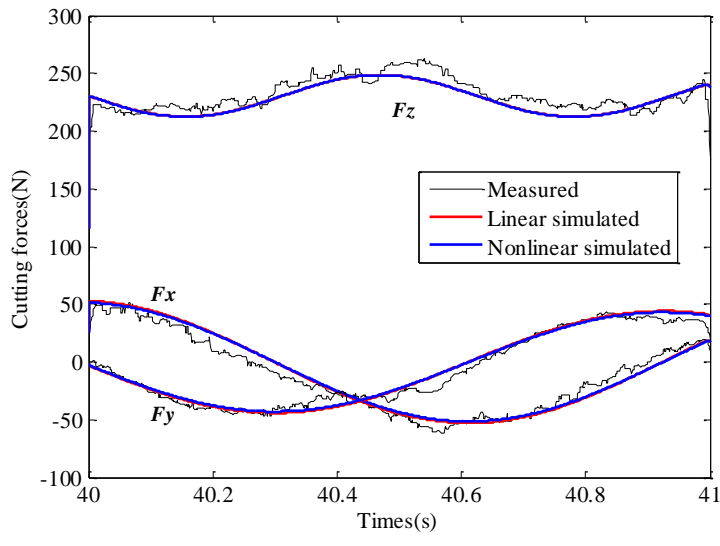

(a)

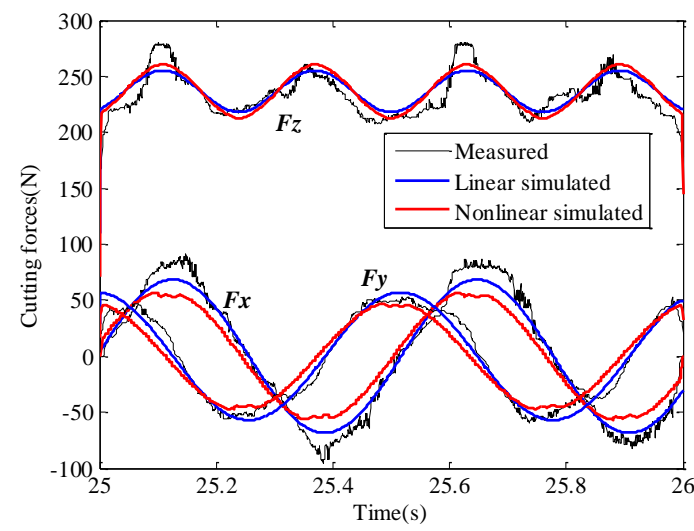

(c)

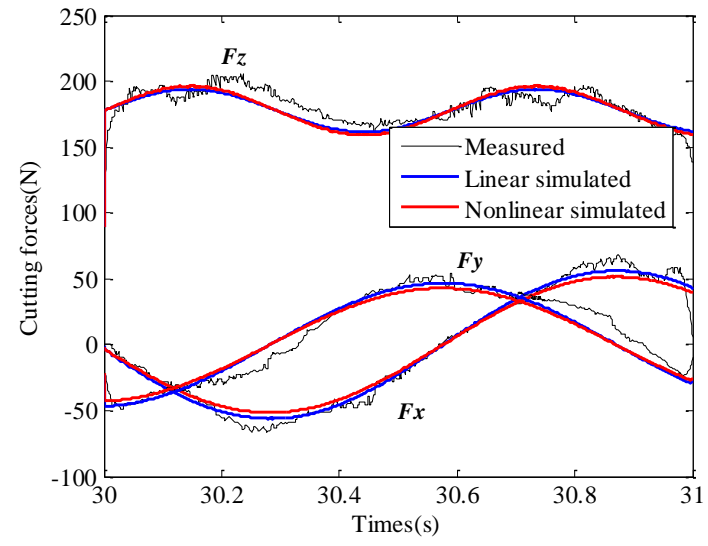

(b)

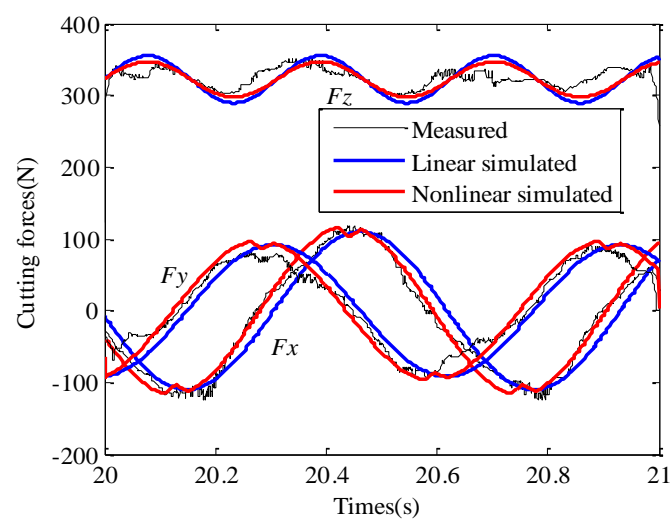

(d)

Fig.7 Comparison of simulated and measured cutting forces

In order to clearly represent the error between linear and nonlinear fitting cutting forces and measured values in Fig.7, the corresponding errors of maximum value for $x, y$ and $z$ directions between simulated and experimental are illustrated in Table 4.

Table 4 Errors of maximum cutting forces

\begin{tabular}{c|c|c|c|c|c|c}
\hline \hline \multirow{2}{*}{ Figures } & \multicolumn{3}{|c|}{ Errors using linear model } & \multicolumn{3}{|c}{ Errors using nonlinear model } \\
\cline { 2 - 7 } & $x$ & $y$ & $z$ & $x$ & $y$ & $z$ \\
\hline Fig.7 (a) & $2.7 \%$ & $2.1 \%$ & $5.6 \%$ & $0.3 \%$ & $0.7 \%$ & $5.5 \%$ \\
Fig.7 (b) & $7.86 \%$ & $13.1 \%$ & $5.5 \%$ & $7.6 \%$ & $12.8 \%$ & $4.3 \%$ \\
Fig.7 (c) & $24.7 \%$ & $8.8 \%$ & $9.1 \%$ & $32.3 \%$ & $9.3 \%$ & $7 \%$ \\
Fig.7 (d) & $6.8 \%$ & $0.7 \%$ & $0.3 \%$ & $2 \%$ & $2.3 \%$ & $2.1 \%$ \\
\hline
\end{tabular}

It can be seen from Table 4 that the error in $z$ direction is relatively small with less than $10 \%$, but to the cutting force in $x$ direction, the error seems a little big in Fig.7(c), experimental forces in $x$ direction fluctuates obviously, it can 
Haiyan Wang, Jianyu Wang, Zhang, Tao and Wu,

Journal of Advanced Mechanical Design, Systems, and Manufacturing, Vol.14, No.1 (2020)

be excluded as gross error. In general, the error values are less than $15 \%$, which can be thought that cutting force model can be used to simulate the change of cutting forces in helical milling of CFRP, at the same time, it can be seen that the nonlinear model does not reflect obvious advantages, the fitting error using linear and nonlinear model under different cutting parameters is almost same. The main reason may be the nonlinear relation between cutting coefficients and cutting parameters are considered whatever linear and nonlinear cutting force model. When the cutting force model is linear, the instantaneous force coefficient method is used to identify the cutting force coefficient, which can be reflected the actual change of cutting force truly with higher fitting precision. But for the nonlinear model, the nonlinear is considered in the model, so the average force coefficient method is able to complete the corresponding simulation.

\section{Conclusions}

1. Based on helical milling principle, cutting forces in helical milling of CFRP are modelled and simulated from linear and nonlinear conditions, and considering the fiber cutting angle of unidirectional CFRP and frontal edges, cutting force model have been modified.

2. It can be known from past experience that the fitting precision of instantaneous force method is better than average force method, and nonlinear model is better than linear model, so cutting force coefficients in the linear model are identified using instantaneous force method and cutting coefficients are identified in the nonlinear model using average force method.

3. In the linear model using instantaneous force method, the cutting coefficients will change with the change of cutting parameters, these coefficients need to be identified with each cutting parameter. The research results can be used to analyze and simulate the cutting forces under different cutting parameters. While in the nonlinear cutting force model, cutting force coefficients are thought as the function of the undeformed chip thickness, cutting force coefficients can be supposed as constant.

4. When considering cutting force coefficients are different in two cases, both models can be used to simulation the cutting force in the helical milling process. No matter linear or nonlinear model, the relationship between cutting force coefficient and cutting parameters need to be correctly considered, thus the changes of cutting force in the helical milling process of composite materials can be simulated relatively accurately.

\section{Acknowledgement}

The authors are grateful for funding supports by the National Natural Science Foundation of China (51705070) and Natural Science Foundation of Hebei (E2014501077) and CSC(China Scholarship Council).

\section{References}

Adem, K.A.M., Fales, R. and El-Gizawy, A.S., Identification of cutting force coefficients for the linear and nonlinear force models in end milling process using average forces and optimization technique methods, International Journal of Advanced Manufacturing Technology, Vol.79 (2015), pp.1671-1687.

Adetoro, O.B. and Wen, P.H., Prediction of mechanistic cutting force coefficients using ALE formulation, International Journal of Advanced Manufacturing Technology, Vol. 46, No. 1-4 (2009), pp.79-90.

Altintas, Y. and Lee, P., A general mechanics and dynamics model for helical end mills, CIRP Annals, Vol.45, No.1 (1996), pp. 59-64.

Azeem, A., Feng, H.Y.and Wang, L.H., Simplified and efficient calibration of a mechanistic cutting force model for ball-end milling, International Journal of Machine Tools and Manufacture, Vol.44 (2004), pp.291-298.

Brinksmeier, E., Fangmann, S. and Rentsch, R., Drilling of composites and resulting surface integrity, CIRP Annals Manufacturing Technology, Vol. 60 (2011), pp.57-60.

Budak, E., Altinas, Y. and Armarego, E.J.A., Prediction of milling force coefficients from orthogonal cutting data, Transactions of the ASME, Vol.118 (1996), pp.216-224.

Gonzalo, O., Beristain, J., Jauregi, H. and Sanz, C., A method for the identification of the specific force coefficients for mechanistic milling simulation, International Journal of Machine Tools and Manufacture, Vol.50 (2010), pp.765774. 
Gonzalo, O., Jauregi, H., Uriarte, L.G. and Lacalle, L.N.L., Predicition of specific force coefficients from a FEM cutting model, International Journal of Advanced Manufacturing Technology, Vol. 43 (2009), pp.348-356.

Gradisek, J., Kalveram, M. and Weinert, K., Mechanistic identification of specific force coefficients for a general end mill, International Journal of Machine Tools and Manufacture, Vol.44 (2004), pp.401-414.

Iyer, R., Koshy, P. and Ng, E., Helical milling: An enabling technology for hard machining precision holes in AISI D2 tool steel, International Journal of Machine Tools and Manufacture, Vol.47 (2007), pp.205-210.

Kalla, D., Ahmad, J.S. and Twomey, J., Prediction of cutting forces in helical end milling fiber reinforced polymers, International Journal of Machine Tools and Manufacture, Vol. 50 (2010), pp. 882-891.

Li, Z.Q., Liu, Q., Ming, X., Wang, X. and Dong, Y., Cutting force prediction and analytical solution of regenerative chatter stability for helical milling operation, International Journal of Advanced Manufacturing Technology, Vol. 73 (2014), pp.433-442.

Liu, C., Wang, G.F. and Dargusch, M.S., Modelling, simulation and experimental investigation of cutting forces during helical milling operations, International Journal of Advanced Manufacturing Technology, Vol.63 (2012), pp. 839850 .

Mongomery, D. and Altintas, Y., Mechanism of cutting force and surface generation in dynamic milling, Journal of Engineering for Industry, Vol.113, No.2 (1991), pp. 160-168.

Pereira, R.B.D., Brandão, L.C., Paiva, A.P., Ferreira, J.R. and Davim, J.P., A review of helical milling process, International Journal of Machine Tools and Manufacture, Vol. 120 (2017), pp. 27-48.

Rey, P.A., LeDref, L., Senatore, J. and Landon, Y., Modelling of cutting forces in orbital drilling of titanium alloy Ti-6Al-4V, International Journal of Machine Tools and Manufacture, Vol. 106 (2016), pp. 75-88.

Sadek, A., Meshreki, M. and Attia, M.H., Characterization and optimization of orbital drilling of woven carbon fiber reinforced epoxy laminates. CIRP Annals - Manufacturing Technology, Vol.61 (2012), pp.123-126.

Schwenzer, M., Obbeler, B.D. and Bergs, T., Comparative study on optimization algorithms for online identification of an instantaneous force model in milling, International Journal of Advanced Manufacturing Technology, Vol.101 (2019), pp.2249-2257.

Shang, S., Qin, X.D., Li, J.H., Li, S.P., Li, H., Huang, T., Jin, Y. and Sun, D., Modelling of cutting forces and researching calibration method in helical milling, International Journal of Advanced Manufacturing Technology, Vol. 94 (2018), pp.2949-2960.

Shirase, K. and Altintas, Y., Cutting force and dimensional surface error generation in peripheral milling with variable pitch helical end mills, International Journal of Machine Tools and Manufacture, Vol.36, No.5 (1996), pp. 567584.

Tlusty, J. and MacNeil, P., Dynamics of cutting forces in end milling, CIRP Annals, Vol.24, No.1(1975), pp. 21-25.

Wan, M., Zhang, W.H., Dang, J.W. and Yang, Y., A novel cutting force modelling method for cylindrical end mill, Applied Mathematical Modelling, Vol.34 (2010), pp. 823-836.

Wang, G.F., Peng, D.B., Qin, X.D. and Cui, Y.H., An improved dynamic milling force coefficients identification method considering edge force, Journal of Mechanical Science and Technology, Vol.26,No.5 (2012), pp.15851590.

Wang, H.Y. and Qin, X.D., A mechanistic model for cutting force in helical milling of carbon fiber reinforced polymers, International Journal of Advanced Manufacturing Technology, Vol. 82 (2016), pp.1485-1494.

Wang, H.Y., Qin, X.D., Ren, C.Z. and Wang, Q., Prediction of cutting forces in helical milling process, International Journal of Advanced Manufacturing Technology, Vol. 58 (2012), pp. 849-859. 